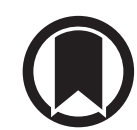

CrossMark

\title{
Awake prone positioning for non-intubated oxygen dependent COVID-19 pneumonia patients
}

\begin{abstract}
To the Editor:
Oxygenation failure recalcitrant to increasing positive end-expiratory pressure is a feature of severe coronavirus disease 2019 (COVID-19) pneumonia [1]. A Chinese group used prone positioning to improve oxygenation for intubated patients with severe COVID-19 pneumonia [2]. However, prone positioning in unconscious patients is labour-intensive and is associated with various complications $[3,4]$. As the incidence of severe COVID-19 pneumonia worldwide increases rapidly, many countries are also facing the problem of diminishing intensive care resources.
\end{abstract}

Prone positioning ventilation is most used today in intensive care units (ICU) for patients with acute respiratory distress syndrome (ARDS) [5-7] and for prevention of ventilator-induced lung injury $[8,9]$. Many mechanisms have been proposed, including relieving the dependent lung regions from the compressive force of the heart's weight [10] or increasing aeration in the originally dorsal lung regions [11]. The overall lung ventilation from dorsal to ventral areas is more homogeneous in the prone position than in the supine position. Prone positioning thus improves oxygenation whilst the other variable, perfusion, remains almost constant in both postures.

The physiological basis behind prone ventilation in an intubated patient with COVID-19 infection should apply to patients breathing spontaneously. However, the literature for prone positioning of non-intubated patients is less available [12], usually described in post-lung transplantation cases [13, 14].

Moreover, the treatment of COVID-19 has mainly been supportive thus far. Trials are still ongoing to validate the use of antiviral and immunomodulatory therapies against this disease, but the use has been limited by the associated adverse events [15]. With the global pandemic putting a strain on many countries' resources, there is an urgency to find a low-risk, low-cost manoeuvre for non-intubated patients that halts disease progression, especially when this has the potential to reduce the need for labour-intensive care and prone ventilation in the ICU.

In our case series, we describe 10 patients with COVID-19 pneumonia requiring oxygen supplementation who underwent awake prone positioning therapy, describing the manoeuvre's effects on patients' oxygenation and outcomes.

Collection of clinical data from infected patients was approved by the Ministry of Health, Singapore, with waiver of written informed consent under the Infectious Disease Act of Singapore. We recruited patients who were admitted to the National Centre for Infectious Diseases since the introduction of this therapy in March 2020. All suitable patients with COVID-19 pneumonia who required oxygen supplementation were started on the protocol. We chose the first 10 patients that were notified to the study team for this series as they were part of the internal audit of the therapy's effectiveness.

The protocol was initiated in a general ward setting. Patients were required to adopt the prone position for $1 \mathrm{~h}$ each session, five sessions per day, each spaced $3 \mathrm{~h}$ apart during waking hours. A patient information sheet was provided to illustrate the possible prone variations. Arms could either be positioned at the side or abducted to less than 90 degrees at the shoulder and flexed at the elbow ("prone superman" posture).

@ERSpublications

Adopting awake prone positioning in non-intubated patients with COVID-19 pneumonia is a lowrisk, low-cost manoeuvre that may potentially delay or reduce the need for intensive care https://bit.ly/ 2LPUnTl

Cite this article as: $\mathrm{Ng} \mathrm{Z}$, Tay WC, Ho CHB. Awake prone positioning for non-intubated oxygen dependent COVID-19 pneumonia patients. Eur Respir J 2020; 56: 2001198 [https://doi.org/10.1183/ 13993003.01198-2020]. 


\begin{tabular}{|c|c|c|c|c|c|c|c|c|c|c|c|c|c|c|c|c|c|c|c|}
\hline \multicolumn{3}{|c|}{ Biographical data } & \multicolumn{3}{|c|}{$\begin{array}{l}\text { Initiation of oxygen } \\
\text { therapy }\end{array}$} & \multicolumn{2}{|c|}{$\begin{array}{l}\text { Awake prone } \\
\text { positioning therapy }\end{array}$} & \multicolumn{6}{|c|}{ Other interventions (day of illness) } & \multicolumn{6}{|c|}{ Outcome measures } \\
\hline 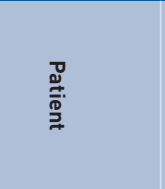 & 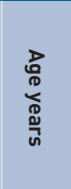 & $\underset{\mathscr{N}}{\stackrel{n}{x}}$ & 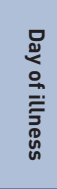 & 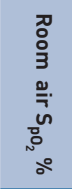 & 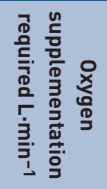 & 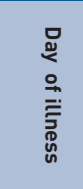 & 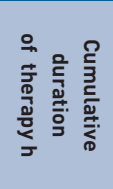 & 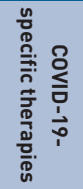 & 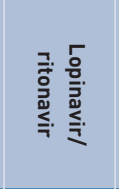 & 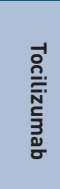 & 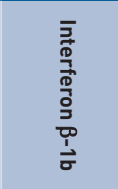 & 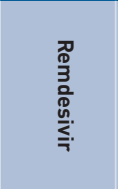 & 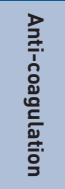 & 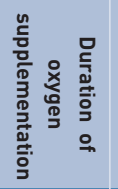 & 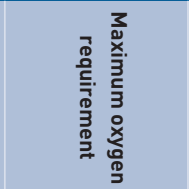 & 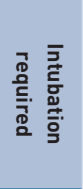 & 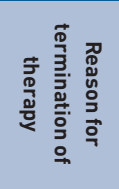 & 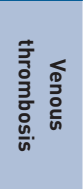 & 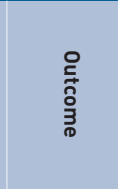 \\
\hline Patient 1 & 59 & M & 13 & 95 & 2 & 13 & 2 & & & & & & No & 2 & NC $2 \mathrm{~L} \cdot \mathrm{min}^{-1}$ & No & Room air & No & Discharged \\
\hline Patient 2 & 66 & $\mathrm{~F}$ & 7 & 88 & 2 & 7 & 32 & Yes & D12-D19 & D11 & & & No & 7 & $\begin{array}{c}\text { HFNC } 60 \mathrm{~L} \cdot \mathrm{min}^{-1}, \\
\mathrm{~F}_{\mathrm{IO}_{2}}, 60 \%\end{array}$ & No & Room air & No & Discharged \\
\hline Patient 3 & 73 & M & 9 & 91 & 3 & 9 & 58 & Yes & D9-D11 & & D10 & & No & 17 & NC $4 \mathrm{~L} \cdot \mathrm{min}^{-1}$ & No & Room air & No & Discharged \\
\hline Patient 4 & 68 & M & 7 & 90 & 3 & 8 & 10 & Yes & & & & D6-D10 & No & 10 & $\begin{array}{l}\text { Intubated, } \\
\mathrm{F}_{\mathrm{IO}_{2}} 100 \%\end{array}$ & Yes & Deceased & No & Deceased \\
\hline Patient 5 & 57 & $\mathrm{~F}$ & 10 & 94 & 1 & 12 & 20 & Yes & & & & D14-D23\# & No & 8 & $\mathrm{VM}, F_{\mathrm{IO}_{2}} 50 \%$ & No & Room air & No & Discharged \\
\hline Patient 6 & 59 & M & 6 & 94 & 2 & 6 & 31 & Yes & & & & D5-D14 & No & 8 & $\mathrm{NC} 2 \mathrm{~L} \cdot \mathrm{min}^{-1}$ & No & Room air & No & Discharged \\
\hline Patient 7 & 55 & M & 9 & 92 & 2 & 10 & 40 & Yes & & & & D10-19\# & No & 9 & $\mathrm{NC} 4 \mathrm{~L} \cdot \mathrm{min}^{-1}$ & No & Room air & No & Discharged \\
\hline Patient 8 & 44 & M & 13 & 91 & 2 & 13 & 15 & & & & & & No & 5 & $\mathrm{NC} 2 \mathrm{~L} \cdot \mathrm{min}^{-1}$ & No & Room air & No & Discharged \\
\hline Patient 9 & 72 & M & 9 & 93 & 2 & 24 & 20 & Yes & D9-D22 & & D11-D22 & & No & 19 & $\mathrm{VM}, F_{\mathrm{IO}_{2}} 50 \%$ & No & Room air & No & Discharged \\
\hline Patient 10 & 53 & M & 16 & 91 & 3 & 16 & 22 & Yes & D10-D14 & & & & Yesף & 4 & NC $3 \mathrm{~L} \cdot \mathrm{min}^{-1}$ & No & Room air & No & Discharged \\
\hline Mean/median+ & 60.0 & & $9^{+}$ & $91.5^{+}$ & $2^{+}$ & $11^{+}$ & $21^{+}$ & & & & & & & $8^{+}$ & & & & & \\
\hline
\end{tabular}

FIGURE 1 Overview of patients who underwent awake prone positioning therapy. \#: these two patients were enrolled in a randomised remdesivir versus placebo trial, with a $50 \%$ chance of receiving remdesivir. "?: for newly diagnosed atrial fibrillation; the patient was started initially started on enoxaparin and later bridged to apixaban. ${ }^{+}$: median value. $S_{\mathrm{pO}_{2}}$ : peripheral oxygen saturation; COVID-19: coronavirus disease 2019; M: male; F: female; D-D: duration of therapy from day of illness; NC: nasal cannula; HFNC: high flow nasal cannula; VM: Venturi mask; $F_{\mathrm{IO}_{2}}$ : fraction of inspired oxygen. 
The patient's face could be placed on either side and patients were allowed to adjust their position for comfort. Haemodynamics and oxygen saturation were charted at 0,30 and 60 min from the start of each session.

This protocol was disseminated to all attending physicians caring for patients with COVID-19 pneumonia. All patients without contraindications were started on this protocol. Contraindications included patients who were drowsy or uncooperative, or those with ophthalmic (e.g. glaucoma), cervical (e.g. spondylosis) or abdominal pathologies (including pregnancy). Patients who were haemodynamically unstable or required oxygen more than a fraction of inspired oxygen $\left(F_{\mathrm{IO}_{2}}\right)$ of $50 \%$ were instead referred to the ICU team. This protocol was suggested to be terminated once the patient has been weaned to room air for at least $24 \mathrm{~h}$.

Decision to start or terminate awake prone positioning protocol was dictated by the attending physician. The study team was not involved in patient care decision for all the recruited patients.

The mean age of the patients was 60 years old and protocol was initiated on median day 11 from illness onset (figure 1). The patients underwent the protocol for a cumulative median of $21 \mathrm{~h}$, with nine patients successfully weaning off oxygen, requiring a median of 8 days. All 10 patients were able to tolerate the protocol as described, allowing for adjustments for comfort.

Three patients were transferred to the ICU due to increasing oxygen requirements, where one patient was intubated and subsequently died from severe ARDS. For the remaining two patients, the prone protocol was continued in ICU and they did not require intubation. Both patients were successfully weaned off oxygen, although one required interim support with high flow nasal cannula oxygenation.

Eight patients were initiated on COVID-19-specific therapies such as lopinavir/ritonavir (figure 1). None of the patients were initiated on anticoagulation for venous thrombosis prophylaxis. There was no incident of the patients developing any venous thrombosis. One patient was started on anticoagulation for newly diagnosed atrial fibrillation.

Our series shows promising data for early prone positioning in non-intubated patients. We compared our findings with our own centre's data for the first 100 patients diagnosed with COVID-19 pneumonia: 12 out of $20(60 \%)$ patients who required supplemental oxygen were eventually intubated. Our series suggests that only 1 out of 10 patients required intubation.

Most patients also reported an improvement in symptoms, but some patients experienced mild side-effects, such as musculoskeletal discomfort, nausea or vomiting. For patients unable to tolerate prone positioning, we advocated lying in left and right decubitus position for $30 \mathrm{~min}$ each, in view of the bilateral nature of COVID-19 pneumonia. The timings of the prone positioning were also planned at least $1 \mathrm{~h}$ after mealtimes to minimise gastrointestinal side-effects. We did not monitor the ratio of the partial pressure of arterial oxygen to $F_{\mathrm{IO}_{2}}$ in our series, as the majority of our patients were in the general ward setting who did not have an intra-arterial line for frequent arterial blood gas measurements. We are also cognisant that other COVID-19-specific therapies could have modified the disease course as well.

Being a low-risk and logistically easy-to-execute intervention in cooperative patients, this therapy has a high potential for reducing ICU workload burden. In addition, it is especially useful when other COVID-19 specific therapies are either unavailable or precluded by the patient's pre-existing conditions (e.g. liver dysfunction, thrombocytopenia). There was also no increase in adverse events in our series. The risk of complications, as seen in ARDS ventilation [3] or prolonged spine surgeries [4], was also lower as the patients were conscious and could vary their positions for comfort.

With critical care teams globally facing resource depletion, we share preliminary evidence that awake prone positioning can be a low-risk, low-cost manoeuvre which can help patients with COVID-19 pneumonia delay or reduce the need for intensive care. This will offload the resource and manpower burden on healthcare as the number of critically ill patients with COVID-19 pneumonia is expected to increase. Further studies are warranted to confirm our results and to evaluate its clinical relevance, including benefits of extending each awake prone positioning session beyond $1 \mathrm{~h}$ if the patient is able to tolerate.

Ziqin $\mathrm{Ng} \oplus^{1}$, Woo Chiao $\mathrm{Tay}^{2}$ and Choon Heng Benjamin Ho $\oplus^{1}$

${ }^{1}$ Dept of Respiratory and Critical Care Medicine, Tan Tock Seng Hospital, Singapore. ${ }^{2}$ Dept of Internal Medicine, Tan Tock Seng Hospital, Singapore.

Correspondence: Ng Ziqin, Dept of Respiratory and Critical Care Medicine, Tan Tock Seng Hospital, 11 Jalan Tan Tock Seng, Singapore 308433. E-mail: ziqin.ng@mohh.com.sg

Received: 15 April 2020 | Accepted after revision: 20 May 2020 


\section{References}

1 Gattinoni L, Chiumello D, Caironi P, et al. COVID-19 pneumonia: different respiratory treatments for different phenotypes? Intensive Care Med 2020; 46: 1099-1102.

2 Sun Q, Qiu H, Huang M, et al. Lower mortality of COVID-19 by early recognition and intervention: experience from Jiangsu province. Ann Intensive Care 2020; 10: 33.

3 Kallet RH. A comprehensive review of prone position in ARDS. Respir Care 2015; 60: 1660-1687.

4 Kwee MM, Ho Y-H, Rozen WM. The prone position during surgery and its complications: a systematic review and evidence-based guidelines. Int Surg 2015; 100: 292-303.

5 Guérin C, Reignier J, Richard J-C, et al. Prone positioning in severe acute respiratory distress syndrome. $N$ Engl J Med 2013; 368: 2159-2168.

6 Sud S, Friedrich JO, Adhikari NKJ, et al. Effect of prone positioning during mechanical ventilation on mortality among patients with acute respiratory distress syndrome: a systematic review and meta-analysis. Can Med Assoc J 2014; 186: E381-E390.

7 Gattinoni L, Carlesso E, Taccone P, et al. Prone positioning improves survival in severe ARDS: a pathophysiologic review and individual patient meta-analysis. Minerva Anestesiol 2010; 76: 448-454.

8 Slutsky AS, Ranieri VM. Ventilator-induced lung injury. N Engl J Med 2013; 369: 2126-2136.

9 Hess DR. Patient positioning and ventilator-associated pneumonia. Respir Care 2005; 50: 892-899.

10 Albert RK, Hubmayr RD. The prone position eliminates compression of the lungs by the heart. Am J Respir Crit Care Med 2000; 161: 1660-1665.

11 Albert RK, Leasa D, Sanderson M, et al. The prone position improves arterial oxygenation and reduces shunt in oleic-acid-induced acute lung injury. Am Rev Respir Dis 1987; 135: 628-633.

12 Scaravilli V, Grasselli G, Castagna L, et al. Prone positioning improves oxygenation in spontaneously breathing nonintubated patients with hypoxemic acute respiratory failure: a retrospective study. J Crit Care 2015; 30: 1390-1394.

13 Feltracco P, Serra E, Barbieri S, et al. Noninvasive high-frequency percussive ventilation in the prone position after lung transplantation. Transplant Proc 2012; 44: 2016-2021.

14 Feltracco P, Serra E, Barbieri S, et al. Non-invasive ventilation in prone position for refractory hypoxemia after bilateral lung transplantation. Clin Transplant 2009; 23: 748-750.

15 Cao B, Wang Y, Wen D, et al. A trial of lopinavir-ritonavir in adults hospitalized with severe Covid-19. $N$ Engl $J$ Med 2020; 382: 1787-1799. 